\title{
HYPERBOLIC AFFINE HYPERSPHERES
}

\author{
TAKESHI SASAKI
}

\section{Introduction}

A locally strongly convex hypersurface in the affine space $\boldsymbol{R}^{n+1}$ is called an affine hypersphere if the affine normals (§1) through each point of the hypersurface either all intersect at one point, called its center, or else are all mutually parallel. It is called elliptic, parabolic or hyperbolic according to whether the center is, respectively, on the concave side of the hypersurface, at infinity or on the convex side. This class of hypersurfaces was first studied systematically by W. Blaschke ([1]) in the frame of affine geometry. In his paper [3] E. Calabi redefined it and proposed a problem of determining all complete hyperbolic affine hyperspheres and raised a conjecture that these hypersurfaces are asymptotic to the boundary of a convex cone and every non-degenerate cone $V$ determines a hyperbolic affine hypersphere, asymptotic to the boundary of $V$, uniquely by the value of its mean curvature. He proved this conjecture for homogeneous convex cones under some conditions on the action of the automorphism group of the cone. In $\S 2$ we will prove that this is the case for closed affine hyperspheres relying crucially on theorems of H. Wu ([17]) and S. Y. Cheng-S. T. Yau ([4]) (Theorem 1). A hyperbolic affine hypersphere is identified, through an appropriate Legendre transformation $(\S 1)$, with a bounded convex domain $\Omega$ in $\boldsymbol{R}^{n}(\xi)$ making use of Wu's theorem. Cheng-Yau's theorem says that there exists a unique convex solution for the following equation of Monge-Ampère type

$$
\begin{cases}\operatorname{det}\left(\frac{\partial^{2} u}{\partial \xi_{i} \partial \xi_{j}}\right)=(H u)^{-n-2} & \text { in } \Omega \\ u=0 & \text { on } \partial \Omega .\end{cases}
$$

Using this equation C. Loewner-L. Nirenberg [9] defined a projectively invariant metric of the domain $\Omega$. Then the Legendre transformation is

Received December 16, 1978. 
an isometry with respect to this metric and the affine metric of the hypersphere (Theorem 2).

In $\S 1$ we recall the definition of an affine hypersphere. In $\S 3$ we derive a composition formula of solutions of the equations of type (\#) above (Theorem 3).

In $\S 4$ we restrict ourselves to the homogeneous case. Here the theory of homogeneous convex cones plays a central role. Let $V$ be a nondegenerate open convex cone in $R^{n+1}(x)$ and $V^{\prime}$ be its dual. $A(V)$ means the group of all linear transformations which leave $V$ invariant. Following V. M. Koecher we define the function called the characteristic function of $V$, by the equation $\phi_{V}(x)=\int_{V^{\prime}} e^{-\langle x, \xi\rangle} d \xi$ for every $x \in V,\langle x, \xi\rangle$ being the value of the linear functional $\xi$ at $x$ (Koecher [8], E. B. Vinberg [16]). We denote by $S_{c}$ the level surface of $\phi_{V}:\left\{\phi_{V}(x)=c\right\}$ which is a noncompact submanifold in $V$. It is also known that the Hessian $d^{2} \log \phi_{V}$ defines the metric on $V$. We denote by $\omega$ the induced metric on $S_{c}$. Now we shall assume the cone $V$ is homogeneous under $A(V)$. Then we will prove that $S_{c}$ is a homogeneous hyperbolic affine hypersphere and every such hyperspheres can be obtained in this way (Theorem $4 \mathrm{a}, 4 \mathrm{~b}$ ). We state another composition formula of solutions of equations of type (\#) and see that $\omega$ is identified with the affine metric. We remark also that $S_{c}$ is a global Riemannian symmetric space when $V$ is a self-dual cone (Proposition 6).

The author would like to express his thanks to his colleagues $\mathrm{K}$. Shiga and T. Tsuji and to Professor S. Kobayashi for pleasant discussions with them.

Added in proof. In May, 1979, Professor S. Y. Cheng kindly informed me that he and S. T. Yau also obtained Theorem 1 in this paper independently and announced it in the 1977 Utah conference on partial differential equations.

\section{§1. Proper hyperbolic affine hyperspheres}

First we recall, for completeness, the definition of affine normals after Calabi's paper [3] (cf. H. Flanders [6]). Let $S$ be a hypersurface in $\boldsymbol{R}^{n+1}$ and $f: S \rightarrow \boldsymbol{R}^{n+1}$ be the imbedding of $S$. The imbedding $f$ defines a volume bundle valued quadratic form $G$ on $S$ by the equation

$$
G=\sum_{i, j} \operatorname{det}\left(\frac{\partial^{2} f}{\partial y^{i} \partial y^{j}}, \frac{\partial f}{\partial y^{1}}, \cdots, \frac{\partial f}{\partial y^{n}}\right) d y^{i} d y^{j} \otimes d y^{1} \wedge \cdots \wedge d y^{n}
$$


in terms of local coordinates $\left(y^{1}, \cdots, y^{n}\right)$ of $S$. This is invariant under unimodular affine transformations in $R^{n+1}$. If this quadratic form is supposed to be non-degenerate, it defines a pseudoriemannian structure tensor $g$ with corresponding volume element $d v(g)$, uniquely defined by the equation

$$
G=g \otimes d v(g)
$$

In this paper we assume that the set $S$ is locally strongly convex. Then the tensor $g$ can be chosen to be positive definite choosing the orientation of $S$ so that $G$ is positive valued. With this Riemannian metric, called the affine metric, the affine normal is defined to be the vector $\boldsymbol{n}=\frac{1}{n} \Delta \boldsymbol{f}$, where $\Delta$ is the Laplace-Beltrami operator with respect to $g$. For an affine hypersphere of hyperbolic or elliptic type (called of proper type together) with the center at the origin, $n$ satisfies the equation

$$
\boldsymbol{n}=-H \boldsymbol{f},
$$

where $H$, called the affine mean curvature, is a nonzero constant. Next we explain a non-parametric characterization of an affine hypersphere. Let $\left(x^{1}, \cdots, x^{n+1}\right)$ be a linear coordinate system of $R^{n+1}$ and $\left\{x^{n+1}=f\left(x^{1}, \cdots\right.\right.$, $\left.\left.x^{n}\right)\right\}$ be the representation of $S$ as the graph of a locally strongly convex function $f$ for $x=\left(x^{1}, \cdots, x^{n}\right)$ ranging in a domain $D \subset \boldsymbol{R}^{n}$. Let $\Omega \subset \boldsymbol{R}^{n}\left(\xi_{1}\right.$, $\cdots, \xi_{n}$ ) be the image of $D$ under the locally invertible mapping $\xi=\operatorname{grad} f$ $=\left(f_{1}, \cdots, f_{n}\right)$, where $f_{i}=\frac{\partial f}{\partial x^{i}}$. We define the function $u\left(\xi_{1}, \cdots, \xi_{n}\right)$ on $\Omega$ by the equation

$$
u(\operatorname{grad} f(x))=-f(x)+(x, \operatorname{grad} f(x)),
$$

where (,) is the pairing giving the canonical duality. We recall $u$ the Legendre transform of $f$ and also the domain $\Omega$ the Legendre transform of $S$ with respect to the coordinates $\left(x^{1}, \cdots, x^{n+1}\right)$. In this formulation Calabi proved

(a) The hypersurface $S$ is a proper affine hypersphere with its center at the origin and the affine mean curvature $H$ if $u$ satisfies the equation

$$
\operatorname{det}\left(\frac{\partial^{2} u}{\partial \xi_{i} \partial \xi_{j}}\right)=(H u(\xi))^{-n-2} \text {. }
$$

For the later use we state an easy 
LEMMA 1. If we take another coordinate system $y=\left(y^{1}, \cdots, y^{n+1}\right)$ such that $y=A x$ for $A=\left(a_{\beta}^{\alpha}\right) \in S L(n+1, R)$ and assume $v(\eta)$ is the Legendre transform of $y^{n+1}$ with respect to $\left(y^{1}, \cdots, y^{n+1}\right), \eta_{j}=\frac{\partial y^{n+1}}{\partial y^{j}}$. Then

(1) $\xi_{i}=\left(\sum a_{i}^{j} \eta_{j}-a_{i}^{n+1}\right) /\left(a_{n+1}^{n+1}-\sum a_{n+1}^{j} \eta_{j}\right)$

(2) $u(\xi)=v(\eta) /\left(a_{n+1}^{n+1}-\sum a_{n+1}^{j} \eta_{j}\right)$

$$
(1 \leq i, j \leq n)
$$

\section{§2. Construction of hyperbolic affine hyperspheres}

In this section we will give an answer to Calabi's conjecture stated in Introduction owing mainly to $\mathrm{Wu}$ and Cheng-Yau (Theorems A and B). Let $S$ be a closed hyperbolic affine hypersphere with center at the origin and the affine mean curvature $H$. This is complete with respect to the affine metric ([5]). (But the author does not know whether the completeness of the affine metric implies the closedness or not.) It is also complete with respect to the induced metric of the Riemannian metric of $\boldsymbol{R}^{n+1}$. Therefore the hypersurface $S$ is complete, noncompact, orientable, smooth and locally strongly convex. In this situation we have

Theorem A. (Wu [17], R. Sacksteder [14]). Such a surface is the full boundary of some closed convex body and is the graph of a non-negative smooth strictly convex function defined in some hyperplane.

By this theorem the hypersurface $S$ can be written globally as the set $\left\{x^{n+1}=f\left(x^{1}, \cdots, x^{n}\right)\right\}$, where $f$ is a positive smooth strictly convex function on $\left\{x^{n+1}=0\right\}$.

Lemma 2. Let $h(z)$ be a positive smooth strictly convex function on $\boldsymbol{R}$ and $H=\{y=h(z)\}$ be the curve in $\boldsymbol{R}^{2}$. Assume every line through the origin $o$ and the points $p$ in $H$ cannot be tangent to $H$ for all $p$. Then. any half line through o can intersect $H$ at most at one point.

Proof. This is straightforward and its proof is omitted.

Lemma 3. Let $S$ be represented as above. Then the tangent plane at any point of $S$ cannot contain the origin.

Proof. In other words the affine normal is not tangent to $S$. Obviously the normal vector in Euclidean sense at one point in $S$ is proportional to $\boldsymbol{n}_{E}:=\left(\xi_{1}, \cdots, \xi_{n},-1\right), \xi_{i}$ being the coordinate of the Legendre transformation. The affine normal $n$ at that point is $\frac{1}{\rho^{2}}\left(\frac{\partial \rho}{\partial \xi_{1}}, \cdots, \frac{\partial \rho}{\partial \xi_{n}}, \rho\right.$ 
$\left.+\sum_{i=1}^{n} \frac{\partial \rho}{\partial \xi_{i}} \xi_{i}\right)$, where $\rho=\operatorname{det}\left(f_{i j}\right)^{-1 / n+2}$ (see [3], p. 26). Hence $\left\langle\boldsymbol{n}, \boldsymbol{n}_{E}\right\rangle=-1 / \rho$ $\neq 0$, completing the proof.

We say a half line $l$ through $o$ is an asymptotic line of $S$ if there exists an unbounded sequence of points $x_{n}$ in $S$ such that half lines through $x_{n}$ and $o$ tend to $l$. And we say $S$ is asymptotic to the boundary of a convex cone when the boundary is equal to the set of all asymptotic lines of $S$. Then we have

Proposition 1. Let $S_{k}=\left\{k p \in \boldsymbol{R}^{n+1} ; p \in S\right\}$. Then $S_{k} \cap S_{k^{\prime}}=\varnothing$ for $k \neq k^{\prime}$ and $V:=\cup_{k>0} S_{k}$ is an open non-degenerate convex cone. $S$ is asymptotic to the boundary of $V$.

Proof. A convex cone is said to be non-degenerate when it contains. no straight line. Assume $S_{k} \cap S_{k^{\prime}} \neq \varnothing$ for some $k \neq k^{\prime}$. This means that there exists a point $p$ in $S_{k}$ such that $\left(k / k^{\prime}\right) p$ is also a point in $S_{k}$. But this cannot occur by the above Lemmas.

Next we shall study the Legendre transform of $S$. Let $C$ be the convex body $\left\{x^{n+1} \geq f(x)\right\} . \quad S$ is the boundary of $C$. For any convex body $K$, the set of all vectors $y \in R^{n+1}$ such that $K+y \subset K$ is called the recession cone of $K$ ([11], p. 61) and denoted by $0^{+} K$. Proposition 1 says that $0^{+} C=\bar{V}$. By definition, the barrier cone $B(K)$ of $K$ is the set of all vectors $\xi$ such that, for some $\beta \in R,\langle x, \xi\rangle \leq \beta$ for every $x \in K$. This is equal to the closure of the set consisting of all outer normals to $K$ if $K$ has interior. Also by definition, the polar cone $W^{0}$ of a convex cone $W$ is the set $\left\{\xi \in \boldsymbol{R}^{n+1} ;\langle x, \xi\rangle \leq 0\right.$ for all $\left.x \in W\right\} . W^{0}$ is the minus of the dual cone $W^{\prime}: W^{0}=\left\{-\xi ; \xi \in W^{\prime}\right\}$. It is known that $B(K)^{0}=0^{+} K([11]$, p. 123). Then we have

Proposition 2. The Legendre transform $\Omega$ of $S$ is the interior of the section of $V^{0}$ with the hyperplane $\left\{\xi_{n+1}=-1\right\}$. The Legendre transform. function $u$ tends to 0 at the boundary of $\Omega$.

Proof. By definition, $\Omega$ can be identified with the set of outer normals $\boldsymbol{n}_{E}$ to $S$, i.e. $\Omega=\operatorname{int}(B(C)) \cap\left\{\xi_{n+1}=-1\right\}=\operatorname{int}\left(V^{0}\right) \cap\left\{\xi_{n+1}=-1\right\}$. By the strong convexity of $f$, we see that $u(\xi)=\sup \{\langle y, \xi\rangle ; y \in S\}$ for $\xi \in \Omega$. Since the condition $\xi_{0} \in \partial \Omega$ is $\sup \left\{\left\langle y, \xi_{0}\right\rangle ; y \in S\right\}=0$, for any sequence $\xi_{i} \in \Omega$ tending to $\xi_{0}$, we have $\lim u\left(\xi_{i}\right)=0$.

From (a) and Proposition 2 it follows that $u$ satisfies the following 
equation of Monge-Ampère type

$$
\begin{cases}\operatorname{det}\left(\frac{\partial^{2} u}{\partial \xi_{i} \partial \xi_{j}}\right)=(H u)^{-n-2} & \text { in } \Omega \\ u=0 & \text { on } \partial \Omega .\end{cases}
$$

Due to the way of the choice of coordinates $u$ is non-positive and $\Omega$ is a bounded convex domain.

Example 1. Put $S=\left\{x \in R^{n+1} ; x^{1} \cdots x^{n+1}=1, x^{1}>0, \cdots, x^{n+1}>0\right\} . \quad S$ is a hyperbolic affine hypersphere ([3]). It is asymptotic to the boundary of the quadrant $V=\left\{x^{1} \geq 0, \cdots, x^{n+1} \geq 0\right\}$. The Legendre transform $\Omega$ is projectively equivalent to an $n$-dimensional simplex which is the bounded intersection of $V^{0}$ with a hyperplane.

Conversely we have the following

Theorem B (Cheng-Yau [4]). Suppose $\Omega$ is a bounded convex domain. Then there exists a unique continuous convex function $u$ on $\bar{\Omega}$ satisfying (\#) such that $\left.u\right|_{\Omega} \in C^{\infty}(\Omega)$.

By direct calculations we have

Lemma $1^{\prime}$. Let $A \in S L(n+1, R)$ be a projective transformation which sends $\Omega$ onto $A \Omega$ in the sense of (1) in Lemma 1. Then the solution $u$ and $v$ of equations (\#) on $\Omega$ and $A \Omega$ respectively are related with each other by the equation (2) in Lemma 1.

Now for a given convex domain $\Omega$ we will consider the convex solution $u$ of the equation (\#) whose existence is assured by Theorem $B$. $u$ is negative by the convexity of $u$. Defining $x^{i}=\frac{\partial u}{\partial \xi_{i}}(1 \leq i \leq n)$, the Legendre transform function $f(x)$ of $u$ with respect to $\xi$ is equal to $-u(\xi)$ $+\sum_{i=1}^{n} x^{i} \xi_{i}$. We denote by $T(\Omega)$ the Legendre transform of $\Omega$, i.e. the image of the map $x(\xi)$. To show that $T(\Omega)$ is the entire space $R^{n}$ we state the maximum principle for the equation

$$
\operatorname{det}\left(\frac{\partial^{2} u}{\partial \xi_{i} \partial \xi_{j}}\right)=(H u)^{-n-2}
$$

on a domain $D$, where $H$ is a negative constant.

LEMma 4. Let $u_{1}$ and $u_{2}$ be two convex functions on $\bar{D}$ satisfying (\#) 
on D. Suppose $u_{1} \geq u_{2}$ on the boundary $\partial D$. Then $u_{1} \geq u_{2}$ on $\bar{D}$.

Proof. This is proved in [4], Proposition 2. (cf. [10]).

We define the function $F$ on $R^{n}$ by $F(x)=\sup \{-u(\xi)+(x, \xi) ; \xi \in \bar{\Omega}\}$. $F(x)=f(x)$ on $T(\Omega)$. If $F(x)$ is equal to $-u(\xi)+(x, \xi)$ for an interior point $\xi$ of $\Omega, x$ belongs to $T(\Omega)$. Here we assume there exists a point $x$ such that $F(x)=-u\left(\xi_{0}\right)+\left(x, \xi_{0}\right)=: d$ for $\xi_{0} \in \partial \Omega$ and $F(x)>-u(\xi)+(x, \xi)$ for $\xi \in \Omega$. Since $\Omega$ is convex we can find a closed simplex $K$ contained in $\Omega$ such that $\partial K \cap \partial \Omega=\left\{\xi_{0}\right\}$. Let $u_{K}$ be the solution of (\#) in $K$. By Lemma 4 we have $u_{K} \geq u$ on $\bar{K}$. Hence $u_{K}(\xi)>(x, \xi)-d$ for $\xi \in \operatorname{int}(K)$ and $u_{K}\left(\xi_{0}\right)=\left(x, \xi_{0}\right)-d$. That is, $-u_{K}(\xi)+(x, \xi)$ attains its maximum value at the boundary point $\xi_{0}$ of $K$. But this is a contradiction, because the Legendre transform of int $(K)$ with respect to $u_{K}$ is the entire space as in Example 1. Therefore we conclude that $T(\Omega)=\boldsymbol{R}^{n}$. Thus the function $f(x)$ is defined for all $x \in \boldsymbol{R}^{n}$ and the hypersurface $\left\{x^{n+1}=f(x)\right\}$ is closed. By (a), this hypersurface is a hyperbolic affine hypersphere with center at the origin. Reversing the above argument constructing a convex domain from an affine hypersphere, we easily see that this hypersphere is contained in $V_{\Omega}$ and asymptotic to the boundary of $V_{\Omega}$, where $V_{\Omega}$ is the polar cone of the non-degenerate convex cone generated by $\Omega$ thinking $\Omega$ as lying in the plane $\left\{\xi_{n+1}=-1\right\}$.

Let $\Omega^{\prime}$ be a projective transform of $\Omega: \Omega^{\prime}=A \Omega$ for $A \in S L(n+1, R)$. Then, by Lemma 1 and Lemma $1^{\prime}$, we see that $V_{\Omega}$ and $V_{\Omega^{\prime}}$ are affinely related by the transformation $\left({ }^{t} A\right)^{-1}$ and the corresponding hyperspheres are also transformed to each other by $\left({ }^{t} A\right)^{-1}$. Therefore, for a given convex cone $V$, we correspond a unique hypersphere irrespective of the choice of the domain $\Omega$ in $V^{0}$.

Hence we have the following

TheOREM 1. Every closed hyperbolic affine hypersphere is asymptotic to the boundary of a convex cone. Conversely, every non-degenerate cone $V$ determines a hyperbolic affine hypersphere asymptotic to the boundary of $V$, and uniquely determined by the value of its mean curvature.

Proof. The uniqueness part is due to the uniqueness assertion of Theorem B and that the affine hyperspheres $S$ and $S_{k}=\{k p ; p \in S\}(k \neq 1)$ can be distinguished from each other by the value of mean curvatures.

Lastly in this section, we consider the metric defined by LoewnerNirenberg [9]. On a bounded convex domain $\Omega$ in $\boldsymbol{R}^{n}(\xi)$ let us take into. 
consideration again the equation (\#). For a negative convex solution $u$ of $(\#)$, they defined the metric

$$
\tau=\frac{1}{H u} d^{2} u
$$

which we call shortly the LN-metric of $\Omega$ in this paper. Such a metric always exists and is uniquely determined by Theorem B. This metric has the projective invariance in the following sense (cf. Lemmas 1 and $1^{\prime}$ ).

(b) Let $A \in S L(n+1, R)$ be a projective transformation which sends $\Omega$ onto $A \Omega$. Then $A$ is an isometry with respect to LN-metrics of $\Omega$ and $A \Omega$.

THEOREM 2. Legendre transformation is an isometry with respect to the LN-metric $\tau$ and the affine metric $g$.

Proof. Let $x^{n+1}=f\left(x^{1}, \cdots, x^{n}\right)$ be an equation of a hyperbolic affine hypersphere. Since $\operatorname{det}\left(f_{i j}, f_{1}, \cdots, f_{n}\right)=f_{i j}$, the affine metric is written as $g=\left(\operatorname{det}\left(f_{i j}\right)\right)^{-1 / n+2} d^{2} f$. But $d^{2} f(x)=d^{2} u(\xi)$ at the corresponding points $x$ and $\xi$ by the Legendre transformation. Hence $\tau=\frac{1}{H u} d^{2} u=\rho d^{2} u=g$.

Remark 1. By the unpublished work of Cheng-Yau [5] the affine metric $g$ is complete. Hence, by Theorems 1 and 2, $\tau$ is also complete.

We will give an example.

EXAMPLe 2. Let $V$ be a circular cone $C(n):=\left\{y_{n+1}>\left(\sum_{i=1}^{n} y_{i}\right)^{1 / 2}\right\}$. Then, by [2], $\phi_{V}(y)=\left(y_{n+1}^{2}-\sum_{i=1}^{n} y_{i}^{2}\right)^{-(n+1) / 2}$ and $S_{1}$ is a hyperboloid of two sheets: $\left\{y_{n+1}^{2}=1+\sum_{i=1}^{n} y_{i}^{2}, y_{n+1}>0\right\}$. On the other hand $u=\sqrt{1-|\xi|^{2}}$, $\xi_{i}=y_{i} / y_{n+1}$. Hence $\Omega$ is the open unit ball. The affine metric is equal to the Hilbert metric of the ball.

\section{§3. A composition formula}

In [3] Calabi has shown a composition formula of two given affine hyperspheres of hyperbolic type constructing a third one of the same type. In this section we will reformulate this composition formula with respect to solutions of the equations of type (\#).

Let $f: S \rightarrow R^{n+1}$ and $g: T \rightarrow R^{m+1}$ be hyperbolic affine hyperspheres with centers at each origin of $\boldsymbol{R}^{n+1}$ or $\boldsymbol{R}^{m+1}$. Then, for any positive constants $c$ and $c^{\prime}$ 
(c) $\boldsymbol{h}:=\left(c e^{t / n+1} f, c^{\prime} e^{-t / m+1} g\right): R \times S \times T \rightarrow \boldsymbol{R}^{n+m+2}$ is another hyperbolic affine hypersphere of dimension greater by one than the sum of dimensions of $S$ and $T([3])$.

Let $V$ (resp. $W$ ) be the cone to whose boundary the hypersphere $S$ (resp. $T$ ) is asymptotic, which is uniquely determined by Theorem 1 . We set $R=\bigcup_{k>0} S_{c^{m+1}} \times T_{c^{\prime} k-(n+1)}$ for $k=e^{t /(n+1)(m+1)}$. By definition of $\boldsymbol{h}$, we have the following

Proposition 3. The hypersphere $\boldsymbol{h}$ is equal to the set $R . \quad R$ is asymptotic to the boundary of the cone $V \times W$.

Hereafter we shall assume $c=c^{\prime}=1$. Let $\left\{x^{n+1}=f(x)\right\}$ and $\left\{y^{m+1}=g(y)\right\}$ be global representations of $S$ and $T$ respectively, where $x=\left(x^{1}, \cdots, x^{n}\right)$ and $\boldsymbol{y}=\left(y^{1}, \cdots, y^{m}\right)$. By $\Omega_{1}$ (resp. $\Omega_{2}$ ) we will denote the Legendre transform of $S$ (resp. $T$ ) with respect to the coordinate $\boldsymbol{x}$ (resp. $\boldsymbol{y}$ ). Also by $u$ (resp. $v$ ) the Legendre transform of $f$ (resp. g). The function $u$ (resp. $v$ ) is the convex solution of the equation (\#) on $\Omega_{1}$ (resp. $\Omega_{2}$ ).

We shall now compute the Legendre transform function for $R$. For that purpose we shall take, as a new coordinate system of $\boldsymbol{R}^{n+m+2}$, the coordinates $(\boldsymbol{\alpha}, \boldsymbol{\beta}, \gamma, \delta)$ defined by $\boldsymbol{\alpha}=\left(\alpha^{1}, \cdots, \alpha^{n}\right)=\boldsymbol{x}, \boldsymbol{\beta}=\left(\beta^{1}, \cdots, \beta^{m}\right)=\boldsymbol{y}$, $\gamma=(n+1) x^{n+1}-(m+1) y^{m+1}$ and $\delta=(n+1) x^{n+1}+(m+1) y^{m+1}$. Then the hypersphere $R$ is expressed by the equations

$$
\begin{aligned}
& \boldsymbol{\alpha}=e^{t / n+1} \boldsymbol{x}, \quad \beta=e^{-t / m+1} \boldsymbol{y}, \\
& \gamma=(n+1) e^{t / n+1} f(\boldsymbol{x})-(m+1) e^{-t / m+1} g(\boldsymbol{y}), \\
& \delta=(n+1) e^{t / n+1} f(\boldsymbol{x})+(m+1) e^{-t / m+1} g(\boldsymbol{y}) .
\end{aligned}
$$

Here we regard $(\boldsymbol{x}, \boldsymbol{y}, t)$ as the coordinates of $R$. Also $(\alpha, \beta, \gamma)$ can be regarded as the coordinates of $R$ since the mapping $(x, y, t) \rightarrow(\alpha, \beta, \gamma)$ is non-singular. Direct calculation leads to the jacobian equation of this mapping:

$$
\begin{aligned}
& d t=-A d \gamma+(n+1) A \sum_{i} f_{i} d \alpha^{i}-(m+1) A \sum_{j} g_{j} d \beta^{j} \\
& d \boldsymbol{x}=e^{-t / n+1} d \boldsymbol{\alpha}+\frac{A}{n+1} \boldsymbol{x} d \gamma-A \boldsymbol{x} \sum_{i} f_{i} d \alpha^{i}+\frac{m+1}{n+1} A \boldsymbol{x} \sum_{j} g_{j} d \beta^{j} \\
& d \boldsymbol{y}=e^{t / m+1} d \beta-\frac{A}{m+1} \boldsymbol{y} d \gamma+\frac{n+1}{m+1} A \boldsymbol{y} \sum_{i} f_{i} d \alpha^{i}-A \boldsymbol{y} \sum_{j} g_{j} d \beta^{j}
\end{aligned}
$$

where $A^{-1}=e^{t / n+1} u+e^{-t / m+1} v . \quad$ Next we set $\xi=\left(\xi_{1}, \cdots, \xi_{n}\right)$ and $\eta=\left(\eta_{1}, \cdots\right.$, 
$\left.\eta_{m}\right)$ by $\xi_{i}=\frac{\partial f}{\partial x^{i}}$ and $\eta_{j}=\frac{\partial g}{\partial y^{j}}$. Also $\rho=\left(\rho_{1}, \cdots, \rho_{n}\right), \sigma=\left(\sigma_{1}, \cdots, \sigma_{m}\right)$ and $\tau$ by $\rho_{i}=\frac{\partial \delta}{\partial \alpha^{i}}, \sigma_{j}=\frac{\partial \delta}{\partial \beta^{j}}$ and $\tau=\frac{\partial \delta}{\partial \gamma}$. Since $\frac{\partial \delta}{\partial t}=e^{t / n+1} f-e^{-t / m+1} g, \frac{\partial \delta}{\partial x^{i}}$ $=(n+1) e^{t / n+1} \xi_{i}$ and $\frac{\partial \delta}{\partial y^{j}}=(m+1) e^{-t / m+1} \eta_{j}$ we have

$$
\begin{aligned}
& \tau=A\left(e^{t / n+1} u-e^{-t / m+1} v\right) \\
& \rho=(n+1)(1-\tau) \xi, \\
& \sigma=(m+1)(1-\tau) \eta .
\end{aligned}
$$

By these equations the Legendre transform function $w$ of $\delta$ with respect to $(\alpha, \beta, \gamma)$ is equal to

$$
w=(n+m+2) A e^{((1 / n+1)-(1 / m+1)) t} u v .
$$

But by the equation $\tau=A\left(e^{t / n+1} u-e^{-t / m+1} v\right)$ we have $e^{((n+m+2) /(n+1)(m+1)) t}$ $=v(1+\tau) / u(1-\tau)$. This yields

$$
w=-\frac{n+m+2}{2}\left((-1)^{n+m+2}(u(1-\tau))^{n+1}(v(1+\tau))^{m+1}\right)^{1 / n+m+2} .
$$

The value of $\tau$ must belong to the interval $(-1,1)$ because $u$ and $v$ are negative and $t$ varies in $R$. Changing the variables by $\tau^{\prime}=(1-\tau) / 2$, $\rho^{\prime}=2(n+1) \rho$ and $\sigma^{\prime}=2(m+1) \sigma$, and setting again $\tau=\tau^{\prime}, \rho=\rho^{\prime}$ and $\sigma=\sigma^{\prime}$ we have the following

Theorem 3. Let $\Omega$ be the domain $\bigcup_{0<\tau<1} \tau \Omega_{1} \times(1-\tau) \Omega_{2}$ in $R^{n+m+1}(\rho$, $\sigma, \tau)$. Then the convex solution of $(\#)$ on $\Omega$ is given by

$$
w=-\left((-1)^{n+m+2}\left(u\left(\frac{\rho}{\tau}\right) \tau\right)^{n+1}\left(v\left(\frac{\sigma}{1-\tau}\right)(1-\tau)\right)^{m+1}\right)^{1 / n+m+2}
$$

up to a constant multiple, where $u$ and $v$ are the negative convex solutions of $(\#)$ on $\Omega_{1}$ and $\Omega_{2}$ respectively.

This construction remains valid, even if one or two of $n$ and $m$ is zero, considering $\Omega_{i}$ as one point in $R$ and $u$ or $v$ as a nonzero constant function.

Example 3. Let $K$ be a simplex in $R^{n}$ defined by inequalities $l_{1} \geq 0$, $\cdots, l_{n+1} \geq 0$, where $l_{i}$ is a linear function. Then the solution of (\#) on $K$ is equal to a constant multiple of $\left(l_{1} \cdots l_{n+1}\right)^{1 / n+1}$. 


\section{§4. Homogeneous hyperbolic affine hyperspheres}

In this section we will consider homogeneous hyperbolic affine hyperspheres. Let $V$ be a non-degenerate convex cone in $R^{n+1}$. First we recall some properties of the characteristic function $\phi_{V}$ defined in Introduction ([16]).

(d) $\phi_{V}(x)$ tends to infinity when $x$ approaches to any point of the boundary of $V$,

(e) The measure $\phi_{V}(x) d x$ is invariant under $A(V)$, i.e. $\phi_{V}(A x)$ $=\phi_{V}(x) / \operatorname{det} A$ for $A \in A(V)$, and

(f) $\log \phi_{V}$ is convex on $V$. Hence $d^{2} \log \phi_{V}$ defines a metric on $V$.

By (d) and (f) the level surface of $\phi_{V}: S_{c}=\left\{\phi_{V}(x)=c\right\}$ is a noncompact submanifold in $V$ which we call the characteristic surface of $V$. We denote by $\omega_{c}$ the induced metric of $d^{2} \log \phi_{V}$ on $S_{c}$.

1. We shall now assume $V$ is affinely homogeneous. The characteristic surface $S_{c}$ is obviously homogeneous with respect to unimodular elements of $A(V)$. Then we have

THEOREM 4a. Every characteristic surface $S_{c}$ is a complete hyperbolic affine hypersphere with mean curvature $a c^{2 / n+2}$, where $a$ is a negative constant depending only on $V$.

Proof. Let $\psi(x)=\log \phi_{V}(x) . \quad S_{c}=\{\psi=\log c\}$ can be written locally as $\left\{x^{n+1}=f\left(x^{1}, \cdots, x^{n}\right)\right\}$ by a smooth function $f$, the coordinate $\left(x^{1}, \cdots, x^{n+1}\right)$ being chosen such that $\psi_{n+1} \neq 0$. First we calculate the Legendre transform $u$ of $f$. Since $\psi$ is constant on $S_{c}$, we have $f_{i}=-\psi_{i} / \psi_{n+1}$ on $S_{c}$. By the definition, $u=-\left(f \psi_{n+1}+\sum_{i=1}^{n} x^{i} \psi_{i}\right) / \psi_{n+1}$. But, by $(\mathrm{e}), \quad \psi(k x)=\psi(x)$ $-(n+1) \log k$ for any $x \in V$ and every positive constant $k$. Hence $\sum_{\alpha=1}^{n+1} x^{\alpha} \psi_{\alpha}(x)=-(n+1)$. Therefore $u=(n+1) / \psi_{n+1}$. Next consider $\operatorname{det}\left(-\psi_{n+1} f_{i j}\right), 1 \leq i, j \leq n$. This is calculated as follows:

$$
\begin{aligned}
\operatorname{det}\left(-\psi_{n+1} f_{i j}\right) & =\operatorname{det}\left(\psi_{i j}-\frac{\psi_{i n+1} \psi_{j}+\psi_{i} \psi_{j n+1}}{\psi_{n+1}}+\frac{\psi_{i} \psi_{j} \psi_{n+1 n+1}}{\psi_{n+1}^{2}}\right) \\
& =\frac{1}{\psi_{n+1}^{2}}\left|\begin{array}{lll}
\psi_{i j} & \psi_{i n+1} & \psi_{i} \\
\psi_{n+1 j} & \psi_{n+1 n+1} & \psi_{n+1} \\
\psi_{j} & \psi_{n+1} & 0
\end{array}\right| .
\end{aligned}
$$

Setting 


$$
\Phi(x)=\left|\begin{array}{cc}
\psi_{\alpha \beta} & \psi_{\alpha} \\
\psi_{\beta} & 0
\end{array}\right| \quad(1 \leq \alpha, \beta \leq n+1),
$$

we have, by the property (e),

$$
\Phi(x)=(\operatorname{det} A)^{2} \Phi(A x) \quad \text { for } A \in A(V) .
$$

Hence $\Phi(x)=b \phi^{2}(x)$ by the homogeneity for some constant $b$ which depends only on $V$ itself. But this means

$$
\left(\psi_{n+1}\right)^{2} \operatorname{det}\left(-\psi_{n+1} f_{i j}\right)=\Phi(x)=b c^{2}
$$

on $S_{c}$. Since $\operatorname{det}\left(f_{i j}\right)=\operatorname{det}\left(\frac{\partial^{2} u}{\partial \xi_{i} \partial \xi_{j}}\right)^{-1}$, we have finally

$$
\operatorname{det}\left(\frac{\partial^{2} u}{\partial \xi_{i} \partial \xi_{j}}\right)=(-(n+1) / u)^{n+2} / b c^{2} \text {. }
$$

By (a), $S_{c}$ is an affine hypersphere with the affine mean curvature $a c^{2 / n+2}$, where $a$ is a negative constant since $S_{c}$ is hyperbolic.

Remark 2. The Legendre transform function $u$ is equal to $(n+1) / \psi_{n+1}$.

Next we shall assume $S$ is a complete hyperbolic affine hypersphere with its center at the origin which is homogeneous under the subgroup $G$ of the unimodular group. By Theorem 1 we can construct a convex cone $V$ to whose boundary the hypersurface $S$ is asymptotic: $V=\bigcup_{k>0} S_{k}$. Let $\tilde{G}:=G \times R^{+}$. The element $g=(g, t) \in \tilde{G}$ acts on $V$ by $g(x)=t \cdot \underline{g}(x)$. $V$ is homogeneous under $\tilde{G}$.

Theorem 4b. Let $S$ and $V$ be as above. Then $S$ is a characteristic surface of $V$.

Proof. We define a function $\chi$ on $V$ by the equation

$$
\chi(x)=k^{-n-1} \quad \text { for } x \in S_{k} .
$$

Since $S_{k} \cap S_{k^{\prime}}=\phi$ for $k \neq k^{\prime}, \chi$ is well defined. Then $\chi(A x)=\chi(x) / \operatorname{det} A$ for $A \in \tilde{G}$. Therefore, by the homogeneity, we have

$$
\chi=b \phi_{V}
$$

for some nonzero constant $b$. This proves Theorem $4 \mathrm{~b}$.

Remark 3. By Theorems $4 \mathrm{a}$ and $4 \mathrm{~b}$ we see that the classification of homogeneous hyperbolic affine hyperspheres is reduced to the classifica- 
tion of homogeneous convex cones. The latter is treated in O. S. Rothaus [13], Vinberg [16] and S. Kaneyuki-T. Tsuji [7], [15]. For instance, we know an inductive method producing all homogeneous convex cones [16]: from a given convex cone $V_{1}$ in $R^{n+1}(x)$ one can construct another homogeneous cone $V$ in $\boldsymbol{R}(t) \times \boldsymbol{R}^{m}(y) \times \boldsymbol{R}^{n+1}(x)$ by the equation $V=\left\{(t, y, x) ; t>^{t} y h^{-1}(x) y\right\}$, where $h$ is a linear mapping on $\boldsymbol{R}^{n+1}$ whose values are real symmetric positive-definite matrices of order $m$ and, corresponding to each element $B$ of some transitive subgroup of $A\left(V_{1}\right)$, there exists a matrix $A \in G L(m, R)$ such that ${ }^{t} A h(x) A=h(B x)$. This method can be transposed to our case to obtain all projectively homogeneous bounded convex domains or all homogeneous hyperbolic affine hyperspheres.

Remark 4. In an inhomogeneous case the level surface is not necessarily an affine hypersphere. For example, let $V=\left\{(x, y, z) \in \boldsymbol{R}^{3} ;|x|<z\right.$, $|y|<z\}$. Then $\phi_{V}=8 z /\left(z^{2}-x^{2}\right)\left(z^{2}-y^{2}\right)$ and $\left\{\phi_{V}=c\right\}$ is not an affine hypersphere, because the function $\Phi$ in the proof of Theorem 4a is not constant on this hypersurface.

2. As for the affine metric we have

Theorem 5. Suppose $V$ is homogeneous. Then the metric $\omega_{c}$ is identified with the affine metric $g$ up to a constant factor.

Proof. Let $\psi$ and $f$ be as in Theorem 4a. Since $d x^{n+1}=-\sum_{i=1}^{n} \frac{\psi_{i}}{\psi_{n+1}} d x^{i}$, we have

$$
\omega_{c}=d^{2} \psi \dot{\mid}_{s_{c}}=\sum_{1 \leq i, j \leq n}-\psi_{n+1} f_{i j} d x^{i} d x^{j}
$$

But $\psi_{n+1}=(n+1) / u$ (Remark 2). Therefore $\omega_{c}=-(n+1) H g$.

Remark 5. The assumption needed to prove Theorem 5 is not the homogeneity of $V$ but the condition that the level surface is an affine hypersphere. (cf. Remark 4).

3. The composition formula in $\S 3$ is, in the homogeneous case, interpreted as follows. The solution $u$ of the equation (\#) is given as a first order logarithmic derivative of the characteristic function, i.e. $u=(n+1) / \psi_{n+1}$ (Remark 2). Since the characteristic function $\phi$ of the product of convex cones $V$ and $W$ is equal to $\phi_{V} \phi_{W}$, the derivative of $\phi$ 
is written using the derivatives of $\phi_{V}$ and $\phi_{W}$. This gives the composition formula for reducible cones.

Associated with the construction of a cone of higher rank (Remark 3) there is another formula of solutions. Let $V_{1}$ and $V$ be the same as in Remark 3. First we note that the dual cone $V^{\prime}$ of $V=\left\{t>{ }^{t} y h^{-1}(x) y\right\}$ is equal to $\left\{(\lambda, \eta, \xi) \in \boldsymbol{R} \times \boldsymbol{R}^{m} \times \boldsymbol{R}^{n+1} ; \lambda \xi-F(\eta, \eta) \in V_{1}^{\prime}\right\}$, where $V_{1}^{\prime}$ is the dual cone of $V_{1}$ and $F(\eta, \eta)$ is a vector function defined by the equation ${ }^{t} F(\eta, \eta) x$ $={ }^{t} \eta h(x) \eta$. Assume the coordinate $\xi=\left(\xi_{1}, \cdots, \xi_{n}, \xi_{n+1}\right)$ is chosen so that $\Omega_{1}=\left\{\xi_{n+1}=1\right\} \cap V_{1}$ is a bounded domain. Then the domain $\Omega=\left\{\lambda+\xi_{n+1}=1\right\}$ $\cap V$ is also bounded and equal to the set

$$
\begin{aligned}
& \left\{\left(\xi_{1}, \cdots, \xi_{n}, \eta, \zeta\right) ;-1<\zeta<1,1-\zeta^{2}-{ }^{t} \eta h_{n+1} \eta>0,\right. \\
& \left.\quad \frac{2(1-\zeta) \xi_{i}+{ }^{t} \eta h_{i} \eta}{1-\zeta^{2}-{ }^{t} \eta h_{n+1} \eta} \in \Omega_{1},(1 \leq i \leq n)\right\}
\end{aligned}
$$

where $\zeta=\xi_{n+1}-1, h_{\alpha}=\frac{\partial h}{\partial x_{\alpha}}$. Next choose the coordinate in $R^{n+1}(x)$ so that $\left\{x^{n+1}=1\right\} \cap V_{1}$ is bounded. Let $\psi^{1}=\log \phi_{V_{1}}, \xi_{i}^{\prime}=-\psi_{i}^{1} / \psi_{n+1}^{1}$ and $u=(n+1) / \psi_{n+1}^{1}$ be the solution of $(\#)$ with respect to $\left(\xi_{1}^{\prime}, \cdots, \xi_{n}^{\prime}\right)$. Setting $k(x)=\sqrt{\operatorname{det} h(x)}$, we have

Proposition 4. The solution $v=v(\xi, \eta, \zeta)$ of the equation (\#) on the domain $-\Omega$ is equal to

$$
\frac{n+m+1}{2} \frac{1-\zeta^{2}-{ }^{t} \eta h_{n+1} \eta}{1-\zeta} \frac{k u}{(n+1) k-k_{n+1} u}
$$

where $k, k_{n+1}$ and $u$ is a function of $\xi^{\prime}$, where $\xi$ and $\xi^{\prime}$ are related by the equation

$$
\frac{2(1-\zeta) \xi_{i}+{ }^{t} \eta h_{i} \eta}{1-\zeta^{2}-{ }^{t} \eta h_{n+1} \eta}=\frac{(n+1) \xi_{i}^{\prime} k+k_{i} u}{(n+1) k-k_{n+1} u}
$$

Proof. The characteristic function of $V$ is known to be equal to $\phi_{V}(t, x, y):=\phi_{V_{1}}(x) / k(x)\left(t-{ }^{t} y h^{-1}(x) y\right)^{1+(m / 2)}([12])$. To calculate its Legendre transform we introduce new variables $z$ and $\tau$, instead of $x^{n+1}$ and $t$, by the equation $z=\left(t+x^{n+1}\right) / 2$ and $\tau=\left(t-x^{n+1}\right) / 2$. Set $\psi=\log \phi_{V}$ and

$$
\xi_{i}=-\psi_{x^{i}} / \psi_{z}, \quad \eta_{j}=-\psi_{y^{j}} / \psi_{z}, \quad \zeta=-\psi_{\tau} / \psi_{z} .
$$

Then, by direct calculation, we have 


$$
\begin{gathered}
1-\zeta=-\frac{1}{\psi_{z}} \frac{m+2}{z+\tau-{ }^{t} y h^{-1} y} \\
1 / \psi_{z}=\frac{1-\zeta^{2}-{ }^{t} \eta h_{n+1} \eta}{2(1-\zeta)} \frac{k u}{(n+1) k-k_{n+1} u}
\end{gathered}
$$

and the relation (3). Noting that $\psi_{z}<0, k_{n+1}<0$ and $u<0$ by the way of the choice of coordinates, we have $1-\zeta>0$ from (4) and $1-\zeta^{2}$ $-{ }^{t} \eta h_{n+1} \eta>0$ from (5), hence $1-\zeta^{2}>0$. Since $k(x)$ is homogeneous of degree $m / 2$ we have $\sum_{\alpha=1}^{n+1} x^{\alpha} k_{\alpha}=\frac{m}{2} k$. Using this relation and the equation $u=-x^{n+1}+\sum_{i=1}^{n} x^{i} \xi_{i}^{\prime}$ we see that $\left((n+1) \xi_{i}^{\prime} k+k_{i} u\right) /\left((n+1) k-k_{n+1} u\right)$ belongs to $-\Omega_{1}$. Therefore $(\xi, \eta, \zeta)$ belongs to $-\Omega$. This means that $(n+m+1) / \psi_{z}$ is the solution of $(\#)$ just on the domain $-\Omega$ and we have proved the proposition.

4. In this subsection we consider the self-dual cone. To formulate another description of the Legendre transformation we introduce the *-mapping due to Koecher [8]. It is a mapping from $V$ to its dual $V^{\prime}$ defined by the equation

$$
\xi=x^{*}=-\left(\psi_{1}(x), \cdots, \psi_{n+1}(x)\right) \quad \text { for } x \in V .
$$

This mapping * has the following properties ([15]).

(g) * sets up a one to one correspondence between $V$ and $V^{\prime}$ and $(A x)^{*}=\left({ }^{t} A\right)^{-1} x^{*}$ holds for every $A \in A(V)$.

(h) If $V$ is homogeneous, then $V^{\prime}$ is also homogeneous and $\phi_{V}(x) \phi_{V^{\prime}}\left(x^{*}\right)$ is constant for all $x$. We denote this constant by $\kappa^{2}$.

In our homogeneous case, by (h), the *-image of $S_{c}$ is also a characteristic surface of $V^{\prime}$ which we denote by $S_{c}^{\prime}$. Taking a hyperplane $H$ such that $U:=H \cap V^{\prime}$ is a non-empty bounded convex domain, we correspond, for every point $x^{*} \in S_{c}^{\prime}$, the intersection point of the line through the origin and $x^{*}$ with $U$. Thus we have a mapping from $S_{c}^{\prime}$ to $U$. And we have easily

Lemma 5. The mapping * followed by this mapping is defined on $S_{c}$ and projectively equivalent to every Legendre transformation of $S_{c}$.

Proposition 5. Suppose $V$ is homogeneous. Then * is an isometry with respect to the metrics $d^{2} \log \phi_{V}$ and $d^{2} \log \phi_{V^{\prime}}$. 
Proof. Let $\psi_{V}=\log \phi_{V}$ and $\psi_{V^{\prime}}=\log \phi_{V^{\prime}}$. By the equation $x^{*}$ $-\operatorname{grad}_{x} \psi_{V}$, we have $d \xi_{i}\left(x^{*}\right)=-\sum_{j} \frac{\partial^{2} \psi_{V}}{\partial x^{i} \partial x^{j}}(x) d x^{j}$. Also $d x^{j}\left(\xi^{*}\right)=$ $-\sum_{k} \frac{\partial^{2} \psi_{V^{\prime}}}{\partial \xi_{j} \partial \xi_{k}}(\xi) d \xi_{k}$. Because ${ }^{* *}=$ identity, we have $\sum_{j} \frac{\partial^{2} \psi_{V}}{\partial x^{i} \partial x^{j}}(x) \cdot \frac{\partial^{2} \psi_{V^{\prime}}}{\partial \xi_{j} \partial \xi_{k}}\left(x^{*}\right)$ $=\delta_{i}^{k}$. Therefore

$$
\begin{aligned}
\left(^{*}\right)^{*}\left(d^{2} \psi_{V^{\prime}}\right) & =\left(^{*}\right)^{*}\left(\sum_{i, j} \frac{\partial^{2} \psi_{V^{\prime}}}{\partial \xi_{i} \partial \xi_{j}} d \xi_{i} d \xi_{j}\right) \\
& =\sum_{i, j, k, l} \frac{\partial^{2} \psi_{V^{\prime}}}{\partial \xi_{i} \partial \xi_{j}}\left(x^{*}\right) \frac{\partial^{2} \psi_{V}}{\partial x^{i} \partial x^{k}}(x) \frac{\partial^{2} \psi_{V}}{\partial x^{j} \partial x^{l}}(x) d x^{k} d x^{l} \\
& =d^{2} \psi_{V} .
\end{aligned}
$$

Now assume $V$ is a self-dual cone. Then $K(x):=-\frac{\partial x^{*}}{\partial x}$ is an automorphism of $V\left([12]\right.$, p. 201) and, moreover, $x^{*}=K(x) x([12]$, p. 192). Let $S=S_{\varepsilon}$. By (h), *iS is an involutive automorphism of $S$. Since $\phi_{V}\left(x^{*}\right)$ $=\phi_{V}(K(x) x)=\phi_{V}(x) / \operatorname{det} K$, we have $\operatorname{det} K(x)=1$ for $x \in S$. Therefore $K(x)$ is an automorphism of $S$ for $x \in S$.

Proposinion 6. If the homogeneous cone $V$ is self-dual, then the characteristic surface $S_{c}$ is a globally symmetric space.

Proof. For one point $x_{0} \in S$ define an automorphism $s$ of $S$ by $s(x)$ $=K\left(x_{0}\right)^{-1} x^{*}$. Since $K(x)$ is symmetric, $s$ is an involution by $(\mathrm{g})$. By the above argument, $s\left(x_{0}\right)=x_{0}$ and $\frac{\partial s}{\partial x}\left(x_{0}\right)=K\left(x_{0}\right)^{-1} \frac{\partial x^{*}}{\partial x}\left(x_{0}\right)=-I$. For a general $S_{c}$, it is enough to translate the symmetry of $S$ to $S_{c}$ by the mapping $S_{c} \ni x \rightarrow \frac{\kappa}{c} x \in S$. Hence we have the proposition.

Example 4. Let $H^{+}(n, K)$ be the cone of positive-definite hermitean symmetric matrices over $K=$ fields $R, C, H$ (quaternions) or the Cayley algebra $\mathrm{Ca}$. Then the followings are the list of all irreducible self-dual cones $V$ and the corresponding globally symmetric spaces $S$.

1. $V=H^{+}(n, R) \quad S=S L(n, R) / S O(n) \quad$ of type AI.

2. $V=H^{+}(n, C) \quad S=S L(n, C) / S U(n)$.

3. $V=H^{+}(n, H) \quad S=S U^{*}(2 n) / S_{p}(n) \quad$ of type AII.

4. $V=C(n) \quad S=S O(1, n-1) / S O(n-1)$ of type BDI.

5. $V=H^{+}(3, C a) \quad S=$ the space of type EIV. 
Remark 6. The author does not know these symmetric spaces are the only ones which can be represented as the affine hyperspheres. If we allow, at the beginning, the metric to be only pseudoriemannian, then some kinds of affine symmetric spaces are realized as the characteristic surfaces. For example: In the space of real symmetric matrices of order $n$, the components of the set $\{$ det $=$ const. $\}$ are such spaces. In fact, they are affine symmetric spaces $S L(n, R) / S O(i, n-i)(0 \leq i \leq n)$.

\section{REFERENCES}

[1] Blaschke, W., Vorlesungen über Differentialgeometrie II, Springer 1923.

[2] Bochner, S., Group invariance of Cauchy's formula in several variables, Ann. of Math., 45 (1944), 686-707.

[ 3 ] Calabi, E., Complete affine hyperspheres I. Symp. Math., 10 (1972), 19-38.

[4] Cheng, S. Y. and Yau, S. T., On the regularity of the Monge-Ampère equation $\operatorname{det}\left(\frac{\partial^{2} x}{\partial x_{i} \partial x_{j}}\right)=F(x, u)$, Comm. on pure and appl. Math., 30 (1977), 41-68.

[5] — Complete affine hypersurfaces, Part I. The completeness of affine metrics, Preprint.

[ 6 ] Flanders, H., Local theory of affine hypersurfaces, J. D'Analyse Math., 15 (1965), 353-387.

[ 7 ] Kaneyuki, S. and Tsuji, T., Classification of homogeneous bounded domains of lower dimension, Nagoya Math. J., 53 (1974), 1-46.

[ 8 ] Koecher, V. M., Positivitätsbereiche im $\boldsymbol{R}^{n}$, Amer. J. Math., 79 (1957), 575-596.

[9 ] Loewner, C. and Nirenberg, L., Partial differential equations invariant under conformal or projective transformations, Contributions to Analysis, 1974, Academic Press, 245-272.

[10] Protter, M. H. and Weinberger, H. F., Maximum principles in differential equations, Prentice-Hall, 1967.

[11] Rockafellar, R. T., Convex analysis, Princeton Univ. Press, 1970.

[12] Rothaus, O. S., Domains of positivity, Abh. Math. Sem. Univ. Hamburg, 24 (1960), 189-235.

[13] — The construction of homogeneous convex cones, Ann. of Math., 83 (1966), $358-376$.

[14] Sacksteder, R., On hypersurfaces with nonnegative sectional curvatures, Amer. J. Math., 82 (1960), 609-630.

[15] Tsuji, T., Classification of homogeneous Siegel domains of type II of dimensions 9 and 10, Proc. of Japan Acad., 49 (1973), 394-396.

[16] Vinberg, E. B., The theory of convex homogeneous cones, Trans. Moscow Math. Soc., 12 (1963), 340-403.

[17] Wu, H., The spherical images of convex hypersurfaces, J. Diff. Geom., 9 (1974), 279-290.

Nagoya University

Current address: Kumamoto University 